\section{Población objetivo del tamizaje de cáncer cervicouterino en el sistema público de atención chileno y su relación con la cobertura de PAP. Implicancias en políticas de salud y asignación de recursos públicos}

\author{
MARÍA TERESA URRUTIA ${ }^{1, a}$, MACARENA GAJARDO $2, b$
}

\section{Factors affecting compliance with PAP smear screening}

Background: PAP smear coverage in Chile has gradually decreased in the last years, from $67 \%$ to $59 \%$, making it necessary to determine the causes of this decline. Aim: To analyze the relationship between the characteristics of the cervical cancer screening target population in the public health care system and the percentage of PAP coverage. Material and Methods: This study was carried out in women aged between 25 and 64 years, belonging to a public health care system and registered in any of the eight primary healthcare centers of a Metropolitan Santiago low income community. The analysis considered information from the recruitment database $(n=6,058)$ and interviewed women database $(n=1,042)$. Results: In $52 \%$ of cases there were difficulties in recruiting women, mainly due to wrong addresses. Among contacted women, $4.1 \%$ had a hysterectomy or had cervical cancer and $1.4 \%$ were dead. When analyzing the variable "adherence to cervical cancer screening" in the interviewed women, $76.8 \%$ reported to comply with the ministerial guidelines. From that group, 20.5\% reported to attend screening at the private health care system. Seventy seven percent of women who had timely screening visits, reported attending screening periodically every 3 years or less. Conclusions: PAP smear coverage must be analyzed considering the different factors that affect it. Among the latter, the exclusion of some women from the target population and performing the screening in private clinics stand out.

(Rev Med Chile 2016; 144: 1553-1560)

Key words: Causality; Confounding Factors; Early Detection of Cancer; Patient Compliance; Uterine Cervical Neoplasms.

Recibido el 28 de julio de 2016 aceptado el 19 de noviembre de 2016.

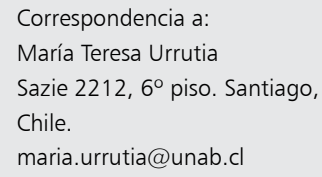

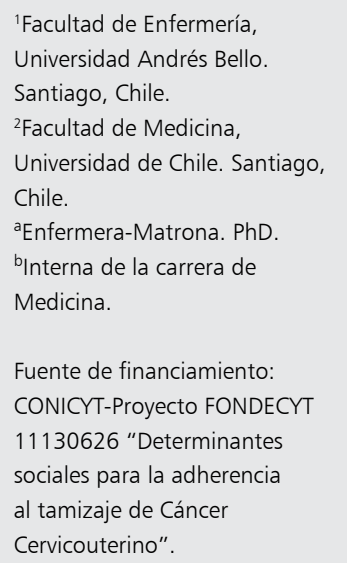

ECYT
ia


población chilena perteneciente al sistema privado y público de salud ${ }^{1}$. La detección precoz de cáncer mediante el PAP, sigue siendo la medida más eficaz y rápida disponible en Chile a nivel público de atención, dado que si se detecta a tiempo puede ser curado en $100 \%{ }^{6}$.

Chile cuenta con 2 estrategias para prevenir el CC, la vacunación contra el virus del papiloma humano (VPH) y la detección temprana de lesiones preinvasoras mediante el PAP. La coexistencia de ambas estrategias es relevante, ya que la vacuna no previene las lesiones preinvasoras ni el cáncer en mujeres ya infectadas ${ }^{7}$.

A pesar de que existe un programa de prevención de CC y que la mortalidad ha disminuido, la cobertura del PAP ha ido en descenso desde 67\% en el año 2008 hasta 59\% en el $2011^{8}$. En el año 2012 el SS Metropolitano Sur-Oriente presentó una cobertura de 54\%, y entre sus comunas, Puente Alto fue una de las tres con las coberturas más bajas, alcanzando $52 \%{ }^{9}$. Cabe señalar que lo ideal para reducir la mortalidad es una cobertura de $80 \%{ }^{10}$, lo que evitaría $3 / 4$ de muertes por $\mathrm{CC}^{11}$; lamentablemente, dicha cobertura nunca ha sido alcanzada en Chile ${ }^{7}$.

La actual situación obliga a encontrar los motivos que expliquen el descenso de cobertura de PAP, sin que exista, hasta el momento, un aumento en la mortalidad ${ }^{12}$. El objetivo de este artículo es analizar la relación entre las características de la población objetivo del tamizaje de CC y el porcentaje de cobertura de PAP, con el propósito de explorar la validez del indicador de cobertura de PAP en dicha comuna.

La presente investigación es parte de un proyecto, cuyo objetivo es explicar la adherencia al tamizaje de CC, a través de una serie de variables no analizadas en el presente artículo.

\section{Material y Método}

El universo corresponde a todas aquellas mujeres entre 25 y 64 años de edad, beneficiarias del sistema de salud público (FONASA) e inscritas en alguno de los 8 consultorios de Puente Alto ( $\mathrm{n}=100.247$ mujeres $)$. La definición de universo en este estudio corresponde a la definición de "población objetivo del tamizaje de CC" en el sistema público de atención, cifra que es utilizada en el denominador para el cálculo de cobertura de PAP.

La muestra fue probabilística estratificada. La estratificación se realizó a nivel de consultorios según el porcentaje de cobertura de PAP, dividiéndolos en cuatro niveles: baja, media-baja, media-alta y alta. Una vez clasificado cada consultorio, se seleccionó al azar un representante de cada nivel. Seleccionados los consultorios, se llevó a cabo el muestreo probabilístico estratificado en cada uno. El tamaño muestral fue calculado para obtener un efecto pequeño de 0,1 , una potencia del $80 \%$, un número de 15 variables latentes y 40 variables observadas con un nivel de confiabilidad del 95\%. Según dicha estimación, se necesitaba al menos entrevistar a 850 mujeres ${ }^{13}$. Con el objetivo de cumplir con este requerimiento y considerando que algunos datos pudieran no ser entregados, se consideró un tamaño muestral cercano a 1.000 mujeres.

Los criterios de exclusión fueron presencia de CC e histerectomía total (HT).

Con el objetivo de contar con una muestra de recambio en caso de no ser ubicada la mujer seleccionada, se elaboró una base de datos de reclutamiento con casos elegidos aleatoriamente ( $\mathrm{n}=6.058$ casos $)$. El reclutamiento fue realizado telefónicamente o mediante visita domiciliaria, logrando un total de 1.042 entrevistas. La aplicación del cuestionario y del consentimiento informado fue entre 40 y 50 minutos. Posterior a ello se resolvieron las dudas de las mujeres.

Se definió "adherencia al tamizaje de CC" como "la realización de al menos un PAP en los últimos tres años"; definición que el sistema de salud utiliza para establecer la cobertura de PAP; dada esta definición, la variable puede tomar dos posibles resultados: (i) Si la mujer adhiere al tamizaje, lo puede hacer en el sistema público o en el privado de atención, (ii) si la mujer no adhiere puede ser porque nunca se ha realizado un PAP o porque el PAP fue hace más de tres años. Para el presente análisis se considera la información de la base de datos de reclutamiento (6.078 casos) y de las mujeres entrevistadas ( 1.042 casos). El estudio cuenta con la aprobación del Comité de Ética del Servicio de Salud Metropolitano Sur-Oriente. Cabe señalar que durante el período de estudio no hubo campañas educativas masivas en torno a este tema.

\section{Resultados}

\section{Base de datos reclutamiento}

De las 6.078 mujeres seleccionadas para reclutamiento, se intentó el contacto con 3.080 mujeres, 


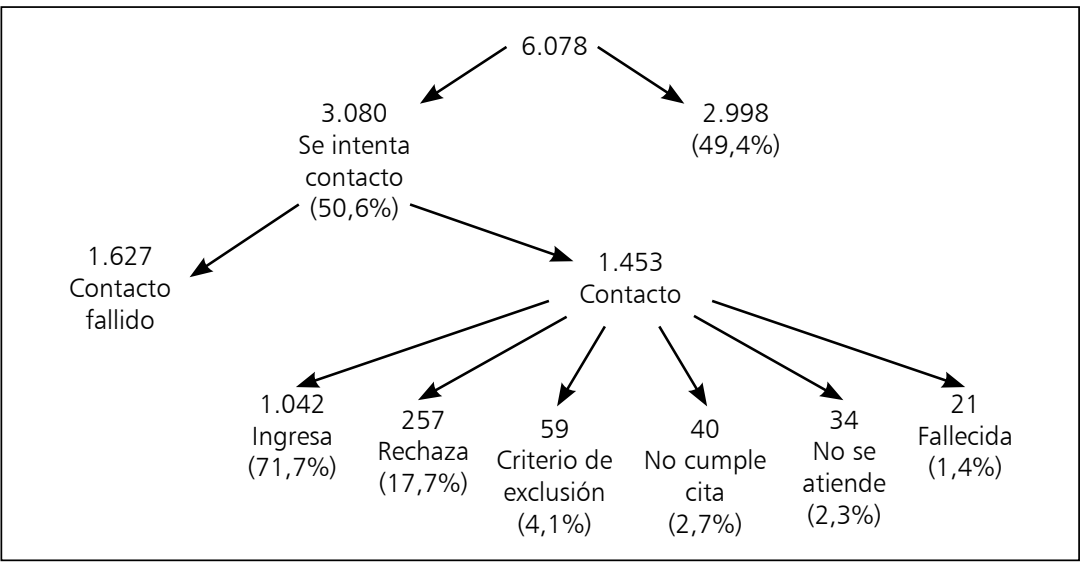

Figura 1. Reclutamiento y resultados del proceso.

Tabla 1. Causas por las cuales las mujeres reclutadas no pudieron ser contactadas

\begin{tabular}{|lcc|}
\hline Causa & Frecuencia & Porcentaje \\
\hline Domicilio visitado no corresponde a la mujer buscada & 849 & $52 \%$ \\
\hline No se encuentra a nadie en el domicilio visitado & 482 & $29,6 \%$ \\
\hline Sin dirección ni teléfono para contactar & 191 & $11,7 \%$ \\
\hline Domicilio visitado es un domicilio antiguo de la mujer & 88 & $5,4 \%$ \\
\hline Domicilio de la base de datos no existe & 17 & $1,1 \%$ \\
\hline Total & 1.627 & $100 \%$ \\
\hline
\end{tabular}

lo que representa el 50,6\% (Figura 1). El resto de las mujeres no fue contactada ya que se logró el tamaño muestral establecido.

En las 3.080 mujeres, el contacto fue fallido en $52,8 \%$ ( 1.627 casos) (Tabla 1). En más de la mitad de ellos fue porque el domicilio no correspondía al de la mujer.

En relación al grupo que se logró contactar $(\mathrm{n}=1.453), 4,1 \%$ cumplía algún criterio de exclusión (Tabla 2 ).

\section{Mujeres entrevistadas}

En relación a las características de las entrevistadas $(n=1.042)$, el promedio de edad fue de 43,31 $\pm 10,83$ años. El promedio de años de escolaridad era de $11,1 \pm 3,2$ años, con un rango entre 0 y 21 . El $18,1 \%$ refería tener título profesional o técnico. El 64,3\% refería estar trabajando de manera remunerada.

El 90,1\% pertenecía a FONASA, 6,3\% a ISAPRE, $0,7 \%(\mathrm{n}=7)$ a las fuerzas armadas, $2 \%$ $(\mathrm{n}=21)$ no tenía previsión y $0,9 \%(\mathrm{n}=9)$ al Pro-
Tabla 2. Situación final de las mujeres contactadas

\begin{tabular}{|lrc|}
\hline Situación & Frecuencia & Porcentaje \\
\hline Ingresa al estudio & 1.042 & $71,7 \%$ \\
\hline Rechaza participar & 257 & $17,7 \%$ \\
\hline $\begin{array}{l}\text { Criterio de exclusión } \\
\text { No cumple la cita agendada }\end{array}$ & 59 & $4,1 \%$ \\
\hline $\begin{array}{l}\text { Refiere no haberse atendido } \\
\text { nunca en el consultorio }\end{array}$ & 34 & $2,7 \%$ \\
Fallecida & 21 & $2,3 \%$ \\
Total & 1.453 & $100 \%$ \\
\hline
\end{tabular}

grama de Reparación y Ayuda Integral en Salud y Derechos (PRAIS). Cabe señalar que, si bien las características del universo consideraba la pertenencia a FONASA, al momento de la entrevista manifestaron pertenecer a otro sistema de salud, motivo que explica el 7\% de mujeres no inscritas en FONASA.

$\mathrm{Al}$ analizar la variable adherencia al tamizaje 
de CC, 76,8\% refirió tener un PAP en los últimos 3 años, por lo tanto, adherente al tamizaje de CC según la norma ministerial chilena. De las mujeres adherentes, 20,5\% refirió realizarlo en el sistema privado de atención. Cabe señalar que, del total de mujeres adherentes, $68 \%$ se lo hizo en el consultorio al cual pertenece (Tabla 3 ).

$\mathrm{Al}$ preguntar la frecuencia con la cual se toma el PAP, $76,9 \%(n=615)$ de las mujeres que tenían el tamizaje al día refirió hacerlo de manera perió- dica cada 3 años o menos (Tabla 4). Si al grupo de mujeres con PAP al día se le suma las que no tienen PAP en los últimos tres años, el porcentaje de mujeres que cumple la norma ministerial baja al 59\% (Tabla 4).

Si se analiza el motivo de toma del examen, 79,7\% refirió que lo hizo por control (Tabla 5). $\mathrm{Al}$ analizar el resultado del último PAP, $89,3 \%$ fue negativo, $3 \%$ positivo $(\mathrm{n}=24)$ y $7,7 \%(\mathrm{n}=62)$ lo desconoce.

Tabla 3. Lugar de toma del PAP en el grupo de mujeres que adhiere al tamizaje $(\mathbf{n}=\mathbf{8 0 0})$

\begin{tabular}{|llcc|}
\hline Sistema de Salud & Lugar último PAP & Frecuencia & Porcentaje \\
Sistema público $79,5 \%$ & Hospital Público & 26 & $3,3 \%$ \\
& Consultorio al cual NO pertenece & 62 & $7,8 \%$ \\
& Consultorio al cual pertenece & 547 & $68,4 \%$ \\
Extra sistema $20,5 \%$ & Centro de salud privado & 122 & $15,3 \%$ \\
& Consulta privada médico/matrona & 24 & $3 \%$ \\
& Cruz Roja & 11 & $1,3 \%$ \\
& Hospital Fuerzas Armadas & 5 & $0,6 \%$ \\
Total & Otro & 3 & $0,3 \%$ \\
\hline
\end{tabular}

Tabla 4. Frecuencia de toma de PAP referida por las mujeres que adhieren al tamizaje

\begin{tabular}{|c|c|c|c|c|}
\hline \multirow[t]{2}{*}{ Frecuencia toma PAP } & \multicolumn{2}{|c|}{ Grupo PAP al día } & \multicolumn{2}{|c|}{ Grupo Total } \\
\hline & Frecuencia & Porcentaje & Frecuencia & Porcentaje \\
\hline Periódicamente cada 3 años & 615 & $76,9 \%$ & & $59 \%$ \\
\hline Irregularmente & 131 & $16,4 \%$ & & $12,6 \%$ \\
\hline Sólo ha tenido 1 PAP & 39 & $4,9 \%$ & & $3,7 \%$ \\
\hline Periódicamente más de 3 años & 9 & $1,1 \%$ & & $0,9 \%$ \\
\hline Sólo ha tenido 2 PAP & 6 & $0,8 \%$ & & $0,6 \%$ \\
\hline Nunca se ha tomado un PAP & & & 34 & $3,3 \%$ \\
\hline PAP hace más de 3 años & & & 208 & $20 \%$ \\
\hline Total & 800 & $100 \%$ & 242 & $100 \%$ \\
\hline
\end{tabular}

Tabla 5. Motivo por el cual se hizo el PAP en el grupo de mujeres con PAP al día

\begin{tabular}{|lcc|}
\hline Motivo & Frecuencia & Porcentaje \\
\hline Por control & 637 & $79,7 \%$ \\
\hline Profesional sugiere que se lo tome & 90 & $11,3 \%$ \\
Consulta por síntomas ginecológicos y profesional toma el PAP & 31 & $3,9 \%$ \\
Se lo tomaron en el control de embarazo & 11 & $1,4 \%$ \\
Porque tiene un PAP previo alterado & 5 & $0,6 \%$ \\
Por campaña de PAP del consultorio/ la llamaron del consultorio & 4 & $0,5 \%$ \\
Otro & 21 & $2,6 \%$ \\
Total & 800 & \\
\hline
\end{tabular}




\section{Discusión}

El presente estudio aporta información importante para explicar, en parte, el descenso de la cobertura de PAP sin que hasta ahora se haya producido un aumento de la mortalidad por CC, ya que, al ser un muestreo representativo, permite extrapolar la información encontrada al universo. Cabe reconocer que una limitación del estudio es que, en el caso de las mujeres entrevistadas, sólo se cuenta con información de aquellas con domicilio conocido.

En relación a la población objetivo del tamizaje, llama la atención que $7 \%$ refiere tener un sistema de salud diferente a FONASA, situación inesperada, dado que es una característica que define la población objetivo. Si esta cifra la aplicamos al total de mujeres consideradas beneficiarias del sistema público en la comuna $(\mathrm{n}=100.247)$, 7.017 mujeres debieran ser excluidas del grupo objetivo (Figura 2), pudiendo ser adherentes o no al tamizaje. Cabe señalar que el porcentaje de mujeres no pertenecientes a FONASA puede ser porque la mujer cambió su sistema de salud sin que haya sido actualizado en la base de datos correspondiente.

Por otro lado, si se agrega el $4,1 \%$ de mujeres con criterios de exclusión y el 1,4\% de fallecidas, da 5,6\% de mujeres que tampoco debieran ser consideradas en el cálculo de cobertura de PAP. Si se hace nuevamente el cálculo y se extrae este porcentaje de la población objetivo de la comuna, un total de 5.613 mujeres debieran ser excluidas. Cabe señalar que la histerectomía es una cirugía frecuente ${ }^{14,15}$, estimándose que $1 / 5$ de las mujeres chilenas será histerectomizada ${ }^{16}$.

Si se analiza el efecto a nivel comunal de los análisis previos, la cobertura podría verse afectada, ya que varía el numerador y el denominador según cada caso. Según lo anterior, si se considera una cobertura comunal de $52 \%$ en un total de 100.247 mujeres, el número de mujeres que adhiere corresponde a 52.128. Al eliminar 5.613 (criterios de exclusión y fallecidas), la población objetivo disminuye a 94.634 mujeres (denominador) y la cobertura sube 3 puntos porcentuales, quedando en $55,1 \%$. Cabe señalar que el numerador no se ve afectado porque estas mujeres no se realizarían el PAP (Tabla 6).

Si el mismo ejercicio se realiza eliminando a las mujeres no beneficiarias de FONASA $(\mathrm{n}=7.017)$ del denominador, la variación en la cobertura de PAP podría incrementar o disminuir según la adherencia de cada una. El rango de variación seria entre $48,4 \%$ (si el 100\% de las mujeres excluidas adhiere al tamizaje) y 55,9\% (si el $100 \%$

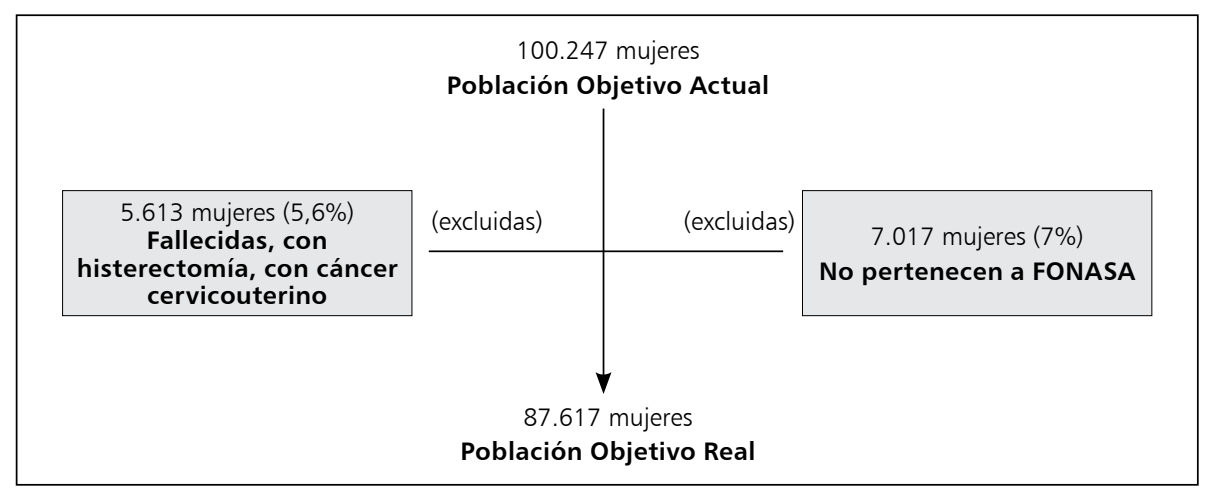

Figura 2. Simulación de población objetivo de la comuna tras exclusión de mujeres por criterios de exclusión y sistema de salud.

Tabla 6. Simulación de cobertura de PAP si se elimina la población de mujeres fallecidas, con cáncer cérvicouterino y con histerectomía total

\begin{tabular}{|c|c|c|c|}
\hline & Fórmula & Población & Cobertura final \\
\hline \multirow[t]{2}{*}{ Situación actual } & Población que adhiere & 52.128 mujeres & \multirow[t]{2}{*}{$52 \%$} \\
\hline & Población total & 100.247 mujeres & \\
\hline \multirow[t]{2}{*}{ Situación simulada } & Población que adhiere & 52.128 mujeres & \multirow[t]{2}{*}{$55,1 \%$} \\
\hline & Población total & 94.634 mujeres & \\
\hline
\end{tabular}


de las mujeres no adhiere al tamizaje) (Tabla 7). Si se suma el 5,6\% que aporta la extracción de mujeres con criterios de exclusión y fallecidas, la cobertura podría variar entre 51,5\% y 59\%, dejando abierta la posibilidad de que las cifras actuales de cobertura de PAP estén bajo de lo real, y por ende ,explicando el no aumento de la mortalidad a pesar de la disminución de cobertura (Tabla 8).

$\mathrm{Al}$ analizar los resultados de adherencia en las entrevistadas, llama la atención el elevado porcentaje de adherencia referido por ellas, distante de la cobertura comunal por más de 24 puntos porcentuales. Cabe señalar que la adherencia al tamizaje es una conducta socialmente esperada, por lo que podría ser una cifra sobreestimada.

A lo anterior se agrega el 20,5\% de mujeres que adhiere en el sistema privado de salud, situación que establece la presencia de una tercera "modalidad de adherencia", es decir, aquel grupo de mujeres considerado población objetivo en el sistema público pero que adhiere al sistema privado de atención. Dicha modalidad se suma a las otras 2 comúnmente consideradas: grupo de mujeres que adhiere al tamizaje y otro que no lo hace.

Las implicancias de una tercera "modalidad de adherencia" tiene efecto directo en el cálculo de cobertura, ya que al 76,8\% de adherencia encontrada en el grupo de entrevistadas, se debiera restar el 20,5\% de mujeres adherentes al sistema privado, dado que actualmente no está siendo considerado adherente al tamizaje. Por lo anterior, el 76,8\% de cobertura desciende automáticamente a 56,3\%, cifra que se acerca bastante a la cobertura comunal actual. A su vez, este hallazgo tiene implicancias en la asignación de recursos en: el per cápita asignado a cada centro de salud, la contratación de personal y la asignación de recursos destinados a campañas educativas y de toma de PAP.

Tabla 7. Simulación de cobertura de PAP si se elimina a las mujeres no beneficiarias de FONASA

\begin{tabular}{|c|c|c|c|}
\hline & Fórmula & Población & Cobertura \\
\hline \multirow[t]{2}{*}{ Situación actual } & Población que adhiere & 52.128 mujeres & \multirow[t]{2}{*}{$52 \%$} \\
\hline & Población total & 100.247 mujeres & \\
\hline \multirow[t]{2}{*}{ Situación simulada* } & Población que adhiere & 45.111 mujeres & \multirow[t]{2}{*}{$48,4 \%$} \\
\hline & Población total & 93.230 mujeres & \\
\hline \multirow[t]{2}{*}{ Situación simulada** } & Población que adhiere & 52.128 mujeres & \multirow[t]{2}{*}{$55,9 \%$} \\
\hline & Población total & 93.230 mujeres & \\
\hline
\end{tabular}

*Si se considera que todas las mujeres no beneficiarias de FONASA adhieren al tamizaje. ${ }^{*}$ Si se considera que ninguna de las mujeres no beneficiarias de FONASA adhieren al tamizaje.

Tabla 8. Comparación de cobertura de PAP simuladas tras la eliminación de mujeres que no cumplen criterios de población objetivo

\begin{tabular}{|c|c|c|c|}
\hline Escenario & Características del indicador & Población & Cobertura \\
\hline \multirow[t]{2}{*}{ Situación actual } & \multirow{2}{*}{$\begin{array}{l}\text { Se mantiene las cifras actuales de población objetivo y población } \\
\text { que adhiere }\end{array}$} & 52.128 mujeres & \multirow[t]{2}{*}{$52 \%$} \\
\hline & & 100.247 mujeres & \\
\hline \multirow[t]{2}{*}{ Escenario 1} & \multirow{2}{*}{$\begin{array}{l}\text { Se eliminan del denominador a las mujeres con cáncer cérvico- } \\
\text { uterino, histerectomía y fallecidas }\end{array}$} & 52.128 mujeres & \multirow[t]{2}{*}{$55,1 \%$} \\
\hline & & 94.634 mujeres & \\
\hline \multirow[t]{2}{*}{ Escenario 2} & \multirow{2}{*}{$\begin{array}{l}\text { Se eliminan del numerador y denominador a las mujeres que } \\
\text { no pertenecen a FONASA (se asume que todas adhieren al PAP) }\end{array}$} & 45.111 mujeres & \multirow[t]{2}{*}{$48,4 \%$} \\
\hline & & 93.230 mujeres & \\
\hline \multirow[t]{2}{*}{ Escenario 3} & \multirow{2}{*}{$\begin{array}{l}\text { Se elimina del denominador a las mujeres que no pertenecen a } \\
\text { FONASA (se asume que ninguna adhiere al PAP) }\end{array}$} & 52.128 mujeres & \multirow[t]{2}{*}{$55,9 \%$} \\
\hline & & 93.230 mujeres & \\
\hline \multirow[t]{2}{*}{ Escenario 4} & \multirow[t]{2}{*}{ Escenario 1 y 2 juntos } & 45.111 mujeres & \multirow[t]{2}{*}{$51,5 \%$} \\
\hline & & 87.617 mujeres & \\
\hline \multirow[t]{2}{*}{ Escenario 5} & \multirow[t]{2}{*}{ Escenario 1 y 3 juntos } & 52.128 mujeres & \multirow[t]{2}{*}{$59,4 \%$} \\
\hline & & 87.617 mujeres & \\
\hline
\end{tabular}


La modalidad de atención identificada nos plantea un símil con el sistema educacional en Chile, donde también se presentan 3 alternativas, siendo la intermedia (educación subvencionada) una alternativa anhelada por la población de bajos recursos. Si bien, esta tercera modalidad de adherencia entrega la tranquilidad de un porcentaje no despreciable de mujeres que aparecen en las estadísticas como NO adherentes, en realidad si lo están haciendo, sin embargo, abre a lo menos 3 interrogantes que debieran ser resueltas en pro de mejorar el programa de prevención de CC.

La primera interrogante es el motivo por el cual $20,5 \%$ de las mujeres que adhieren no eligen el sistema público de atención. La segunda interrogante es la calidad, a la cual ese $20,5 \%$ se está exponiendo, en lo que respecta a la obtención de la muestra citológica como en lo relacionado al análisis e interpretación de la muestra. La tercera interrogante es cómo evitar que esta tercera modalidad de atención afecte las estadísticas de cobertura y la asignación de recursos en salud.

\section{Recomendaciones}

Los resultados de este estudio permiten plantear algunas recomendaciones con el objetivo de mejorar la calidad del programa y sus indicadores.

La actualización de las bases de datos es una de las principales medidas que debiera ser tomada, por su efecto directo en el cálculo de la cobertura. Se hace necesario contar con un sistema que permita entregar información actualizada de la población objetivo del tamizaje, para poder calcular la cobertura lo más cercana a la realidad. Es interesante intentar explicar el porcentaje de direcciones erradas, y, por ende, cuáles son las razones por las cuales la mujer no entrega información correcta. Se hace necesario recomendar que el personal de salud advierta a la mujer sobre la importancia de dicha información, así como también de actualizarla cuando corresponda. Una actualización constante de datos permite confiar en el denominador del indicador en cuestión, excluyendo por ejemplo a las mujeres fallecidas.

A lo anterior se agrega la necesidad de un sistema de datos-registro a nivel nacional, similar al registro de inmunizaciones "el cual permite registrar en cualquier ámbito o establecimientos de la red sanitaria, tanto pública como privada... entregando información oportuna, clara y certera para la toma de decisiones" 17 . Este registro debiera tener información de toda mujer sometida a tamizaje de CC, de los resultados del examen y en caso de tener un PAP alterado, contar con información del diagnóstico y tratamiento. Dicha información debiera ser reportada por el sistema público y por el sistema privado, además de incluir información de mujeres sometidas a histerectomía y defunciones. Esta herramienta "en línea" permitiría retirar oportunamente del denominador a mujeres sin útero y a las diagnosticadas con CC. Por otro lado, este sistema permitiría identificar el $20,5 \%$ de mujeres que en este estudio adhiere en el sistema privado, y que actualmente los profesionales del sistema público desconocen.

En relación a la calidad del PAP, se hace necesario un sistema que permita evaluar periódicamente a los operadores (profesionales que toman la muestra citológica) como a los profesionales que analizan la muestra. "La sensibilidad del examen es la cualidad más importante, ya que este valor es inversamente proporcional al porcentaje de falsos negativos"18. Los falsos positivos o negativos se producen generalmente por no identificar las células anormales o por hacer un diagnóstico incorrecto ${ }^{19}$. Al respecto, la gran ventaja que tiene el sistema público y su respectiva red de laboratorios es el volumen de exámenes analizados y el constante control de calidad de los mismos. Cabe señalar que desde 1993, el Centro de Oncología de la Universidad de Chile, en convenio con el Instituto de Salud Pública, realiza dicho control de calidad el cual incluye evaluar las habilidades diagnósticas individuales de los diferentes profesionales que participan de la citología ginecológica. Dicha exigencia no se extiende al sistema privado de atención ${ }^{20}$. Por otro lado, si una mujer es erróneamente NO diagnosticada, con el tiempo el costo de diagnóstico y tratamiento será significativamente mayor. Esta recomendación incidiría directamente en el grupo de mujeres adherente en el sistema privado de atención, las que en caso de ser diagnosticadas con CC, y dado que el CC es patología GES, serán atendidas con los recursos del sistema público en la mayoría de los casos. Por otro lado, es necesario permanecer cauteloso respecto de las cifras de mortalidad, ya que, si bien puede ser explicado por la adherencia al sistema privado, las consecuencias de dicha adherencia en el largo plazo aún no se conocen. 
Cabe recordar que la progresión de una lesión preinvasora es lenta, por lo que en el caso que una mujer sea diagnosticada erróneamente como sana, no asegurará que eso sea correcto, dada la falta de controles de calidad de laboratorios que procesan dicho examen, así como también del porcentaje de falsos negativos que el PAP ha mostrado ${ }^{19}$.

Finalmente, se hace necesario desarrollar investigaciones que permitan conocer las características de la población denominada en este artículo como tercera modalidad de adherencia. Es importante conocer, además, las causas por las cuales no adhieren al sistema público. Estudios de satisfacción usuaria permitían identificar las falencias en la atención, y, por ende, las medidas para mejorarla.

\section{Referencias}

1. Ministerio de Salud Chile (MINSAL). Programa Nacional de Cáncer Cervicouterino. Citado el 8 de mayo de 2013. Disponible en http://www.minsal.gob.cl/portal/ url/page/minsalcl/g_proteccion/g_programacanc er/ programacancercericouterino.html.

2. Dickinson JA, Stankiewicz A, Popadiuk C, Pogany L, Onysko J, Miller AB. Reduced cervical cancer incidence and mortality in Canada: national data from 1932 to 2006. BMC Public Health 2012; 12: 992.

3. Haider G, Parveen Z, Anjum F, Munir A. Pap smear, an important screening tool to detect precancerous stage of carcinoma of cervix. J Ayub Med Coll Abbottabad 2013; 25 (1-2): 26-7.

4. Kasinpila C, Promthet S, Vatanasapt P, Sasieni P, Parkin DM. Evaluation of the nationwide cervical screening programme in Thailand: a case-control study. J Med Screen 2011; 18 (3): 147-53.

5. Ko MJ, Kim J, Kim Y, Lee YJ, Hong SR, Lee JK. Cost-effectiveness analysis of cervical cancer screening strategies based on the Papanicolaou smear test in Korea. Asian Pac J Cancer Prev 2015; 16 (6): 2317-22.

6. Peirson L, Fitzpatrick-Lewis D, Ciliska D, Warren R. Screening for cervical cancer: a systematic review and meta-analysis. Syst Rev 2013 May 24; 2: 35.

7. Fica A. Prevención del cáncer cérvico-uterino en Chile. Mucha vacuna y poco Papanicolaou. Rev Chilena Infectol 2014; 31 (2): 196-203.

8. Departamento de estadísticas e información en salud. Cobertura. Citado el 8 de mayo de 2013. Disponible en: http://www.deis.cl/cobertura/

9. Programa de Prevención Cáncer Cervicouterino SSM-
SO. Cobertura examen Papanicolaou 2012. Estadísticas. http://www.ssmso.cl/estadisticas/Estadisticas_2012/ Anuario_005.xls

10. Organización Panamericana de la Salud (OPS) Cáncer Cervicouterino. Citado el 10 de diciembre de 2005. Disponible en http://www.paho.org/hq/index.php?option $=$ com_content\&view $=$ category\&layout $=$ blog\&i$\mathrm{d}=3595$ \&Itemid $=3637$ \&lang=es

11. Sociedad Chilena de Obstetricia y Ginecología. Noticias SOCHOG. Citado el 8 de mayo de 2013. Disponible en: http://www.sochog.cl/noticias-sochog.php?id=71

12. Urrutia MT. Cáncer Cervicouterino en Chile: análisis de un nuevo paradigma preventivo. Temas de la agenda pública año $10 / \mathrm{N}^{\circ} 78 /$ mayo de 2015. Disponible en http://politicaspublicas.uc.cl/publicacion/serie-temasde-la-agenda/serie-n-78- cancer-cervicouterino-en-chile-analisis-de-un-nuevo-paradigma-preventivo/

13. Soper DS. (2013). A-priori Sample Size Calculator for Structural Equation Models [Software]. Disponible en http://www.danielsoper.com/statcalc

14. Aarts JW, Nieboer TE, Johnson N, Tavender E, Garry R, Mol BW, et al. Surgical approach to hysterectomy for benign gynaecological disease. Cochrane Database Syst Rev. 2015 Aug 12; (8): CD003677.

15. Sardiñas R. La histerectomía laparoscópica y sus aspectos fundamentales. Revista Cubana de Cirugía 2015; 54 (1) 82-95.

16. Ahumada A, Aliaga P, Bolívar N. Enfoque integral de la paciente histerectomizada. Rev Hosp Clín Universidad de Chile 2001; 12 (3): 229-34.

17. Ministerio de Salud Chile (MINSAL 1). Estrategia digital en salud. Registros nacionales. Citado el 10 de diciembre de 2015. Disponible en http://www.salud-e.cl/category/ proyectos/registros-nacionales/

18. Sánchez L, Rojas A, Riquelme M, Quezada O, López F. Papanicolaou: frotis sin componente endocervical. Rev Chil Obstet Ginecol 2008; 73 (3): 173-8.

19. Gallegos V, Gallegos M, Patricia G, Mota V Escoto S. Cáncer cérvicouterino. Causas de citología no útilRev Latinoamer Patol Clin; 59 (1): 23-7. Disponible en http://www.medigraphic.com/pdfs/patol/pt-2012/ pt121e.pdf

20. Instituto de Salud Pública. Gobierno de Chile. Instructivo PEEC 2016. Citado el 28 de diciembre 2016. Disponible en: http:/www.ispch.cl/sites/default/files/ INSTRUCTIVO_PEEC_2016.pdf

21. Terrazas S, Ibáñez C, Lagos M, Poggi H, Brañes J, Barriga $\mathrm{MI}$, et al. Examen de detección de virus papiloma humano en el tamizaje de Cáncer Cervicouterino en un Servicio de Salud de Santiago, Chile. Rev Med Chile 2015; 143: 56-62. 